\title{
Unilateral pulmonary thromboendarterectomy for iatrogenic pulmonary hypertension in a ten-year-old child
}

\author{
J. Zacharias, FRCS (CTh), S. C. Clark, FRCS (CTh), J. R. L. Hamilton, FRCS, J. H. Dark, FRCS, and A. Hasan, FRCS (CTh), \\ Newcastle upon Tyne, United Kingdom
}

$\mathrm{P}$ leurovenous shunts are inserted for recurrent pleural fluid collections that are resistant to treatment. The limb in the central vein can act as a focus for thrombus formation. A foreign body in the right atrium can accumulate thrombus, and fragments embolize, leading to pulmonary hypertension over a prolonged period. ${ }^{1}$ We present a case of a 10 -year-old boy who had a unilateral pulmonary thrombus 3 years after insertion of a pleurovenous shunt. A pulmonary thromboendarterectomy with cardiopulmonary bypass and profound hypothermic circulatory arrest is the accepted treatment in adults. ${ }^{2}$ In this case we carried out a unilateral thromboendarterectomy, with a very satisfactory result. We also present 5 -year follow-up details regarding this procedure.

\section{Clinical Summary}

The patient was a 10-year-old boy with cystic fibrosis. High-dose pancreatic enzyme supplementation led to fibrosing colonopathy and a lymphoperitoneum requiring laparotomy and drainage in 1993. Later that year, a right-sided pleural effusion was noticed, and nearly $900 \mathrm{~mL}$ of fluid was aspirated. A computed axial tomographic scan did not show mediastinal lymphadenopathy, and lymphangiography demonstrated an obstruction to lymph flow in the lower abdomen. In August 1993, a peritoneoatrial shunt was implanted, with good symptomatic relief. In view of repeated chest aspirations for symptoms, a pleurovenous shunt was placed from the right thorax to the superior vena cava in October 1994. The child was well until September 1997, when a gradual decrease in exercise tolerance and progressive dyspnea were noted. A pulmonary angiogram showed an absence of perfusion to the right lung (Figure 1). In view of the length of symptoms, lytic therapy was believed to be unwarranted, and a decision to carry out a pulmonary thromboendarterectomy was made. At surgical intervention, through a median sternotomy, the right ventricle was noticed to be under strain, and systolic pulmonary artery pressures of $50 \mathrm{~mm} \mathrm{Hg}$ were measured. Full cardiopulmonary bypass was established with a cannula in the inferior vena cava. A thick sleeve of thrombus

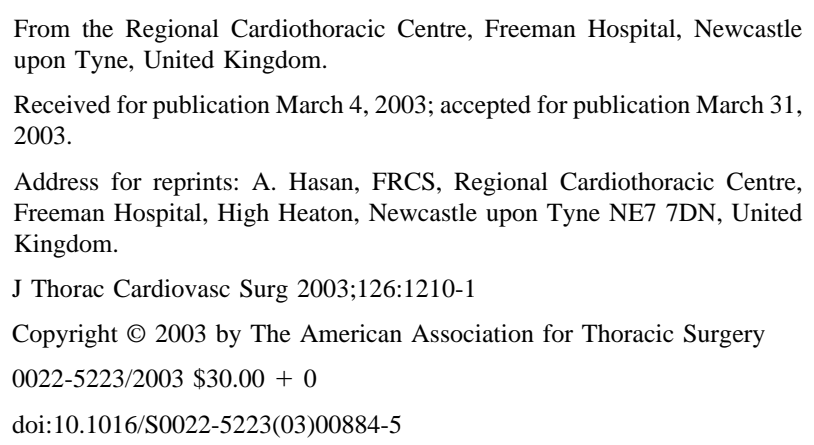

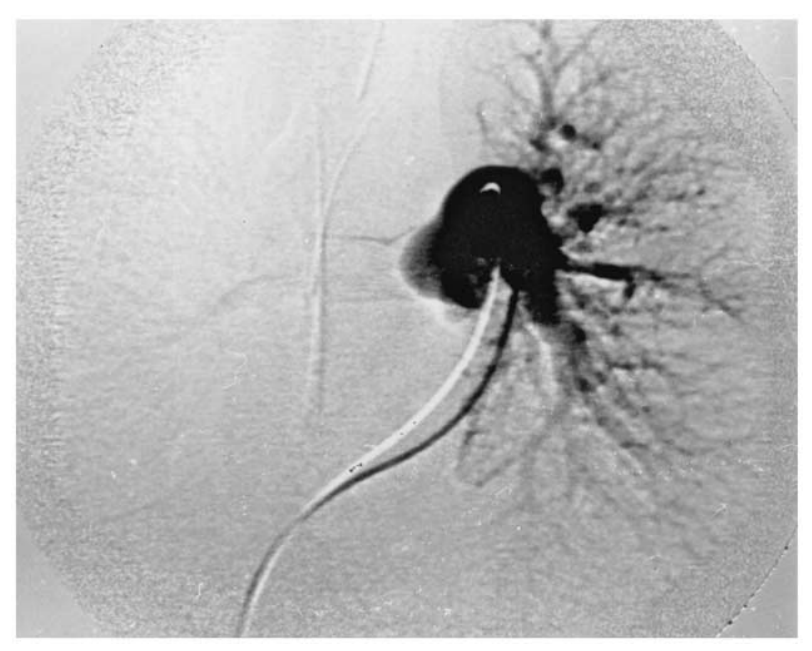

Figure 1. Pulmonary angiogram showing the absence of perfusion to the right lung.

around the pleurovenous shunt precluded cannulation of the superior vena cava. Once bypass was started, the patient was cooled to $18^{\circ} \mathrm{C}$. During cooling, the right pulmonary artery was incised longitudinally, and thrombus was identified. This was mobilized until the branch pulmonary arteries were reached. The circulation was stopped at $18^{\circ} \mathrm{C}$, and further dissection was undertaken to complete the thromboendarterectomy, requiring a short period of circulatory arrest. Perfusion was reestablished, and the right pulmonary artery was closed in 2 layers with 6-0 Prolene sutures (Ethicon, Inc, Somerville, NJ). Exploration of the left pulmonary artery showed no evidence of thrombus.

During a further period of circulatory arrest, the right atrium was explored, and a large $(5 \times 3 \mathrm{~cm})$ thrombus was found pedunculated and adherent to the tubing of the shunt in the atrium. This was excised from the shunt tubing, and all fragments were cleared from the right atrium before reestablishing circulation. Rewarming was undertaken, and once the patient temperature reached $37^{\circ} \mathrm{C}$, cardiopulmonary bypass was easily discontinued. The pulmonary artery pressures measured after the procedure were significantly lower than before $(36 \mathrm{~mm} \mathrm{Hg}$, systolic). Postoperative recovery was uneventful, with normal blood gas levels and a short period on intensive care, after which the patient was discharged home on the fifth day completely asymptomatic. Before discharge, the child was anticoagulated using warfarin.

Five months later, a repeat pulmonary angiogram showed improved perfusion of the right lung fields (Figure 2). The child remains well with good effort tolerance. A follow-up magnetic resonance angiogram 5 years later confirmed good patency of the 


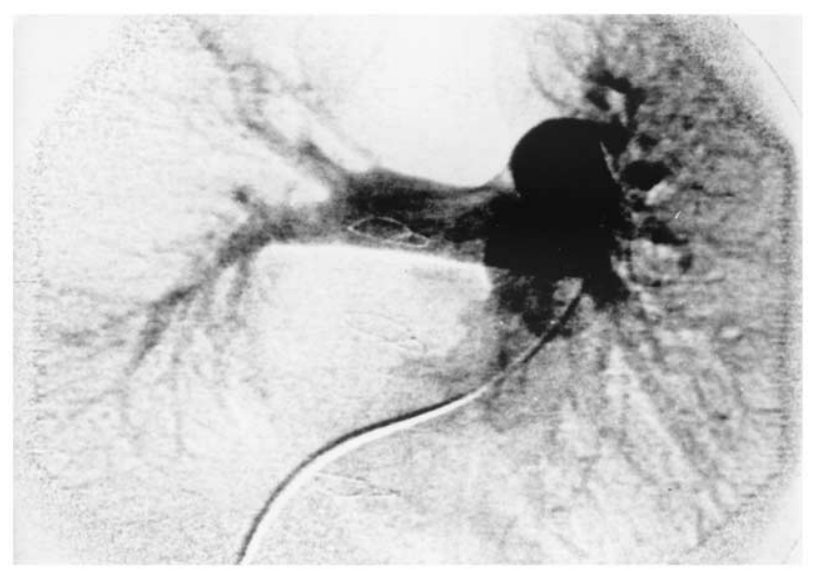

Figure 2. Repeat pulmonary angiogram 5 months after the operation.

right pulmonary artery, with no further progression of disease (Figure 3).

\section{Discussion}

The insertion of a pleurovenous shunt is a useful palliative procedure to achieve symptom control. The complications mentioned in the literature concentrate on common infective and technical problems because they are by far the most common complications. One of the most serious consequences is the development of severe pulmonary hypertension attributed to multiple and recurrent pulmonary embolization arising from the foreign body in the atrium; however, the incidence is exceedingly low. All reports in the literature deal with ventriculoatrial shunts inserted for hydrocephalus into young children with near-normal life spans. The incidence appears to vary between $0.3 \%^{1}$ and $3 \%^{3}$ in different populations. Clinically, the diagnosis is easily missed, and a review of postmortem studies suggests an incidence that might be as high as $59.7 \% .^{1}$ Most insertions of pleurovenous shunts are palliative in nature, and patients have a short life span, not living long enough for pulmonary hypertension to develop. It is also probably underdiagnosed as a result of the nonspecific symptoms.

We believe that oral anticoagulation is indicated in all patients with pleurovenous shunts with a reasonable life expectancy because small emboli can be completely asymptomatic. Initial endothelial damage induced by thrombotic blood components embolizing from the atrium results in an alteration of the pulmonary endothelium, promoting platelet aggregation and thus vascular thrombosis. ${ }^{4}$ This would suggest that even if the source of emboli were removed, the progression of pulmonary vascular disease would continue unless fully anticoagulated. Despite the risks associated with anticoagulation in children, we recommend it to reduce the risk of pulmonary microembolization. The case report by Lambert and colleagues ${ }^{5}$ suggests that an indwelling catheter in

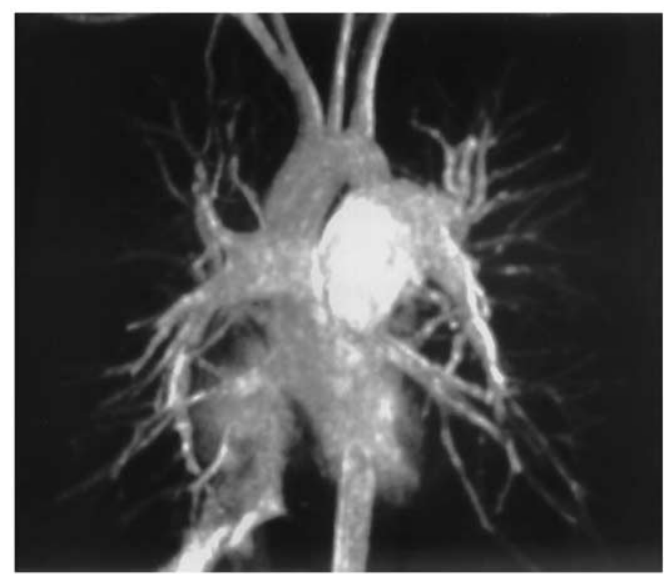

Figure 3. Magnetic resonance angiogram 5 years after the operation showing good flow down the right pulmonary artery.

a central vein might also carry similar risks. There are similarities in the 2 cases in which both patients had unilateral disease arising from a foreign body in a central vein. After administration of warfarin, the results of the operation have been maintained for 5 years after the operation itself.

The procedure of pulmonary thromboendarterectomy in adults is well described by Jamieson and colleagues. ${ }^{2}$ We recommend this operation in the pediatric population in view of the satisfactory result and short period of circulatory arrest it entails. This patient had no disease in the left pulmonary artery, which is unusual, because it is almost always a bilateral disease. ${ }^{2} \mathrm{We}$ find it hard to explain but suspect that the initial incident was probably a large embolus around which further thrombus organization occurred in the weeks before presentation. The operation restored blood flow to the right lung to normal and brought about a resolution of symptoms. The patient was discharged on oral anticoagulation and is well 5 years after surgical intervention.

\section{References}

1. Pascual JM, Prakash UB. Development of pulmonary hypertension after placement of a ventriculoatrial shunt. Mayo Clin Proc. 1993;68:117782.

2. Jamieson SW, Auger WR, Channick RN, Kriett JM, Tarazi RY, et al. Experience and results with 150 pulmonary thromboendarterectomy operations over a 29-month period. J Thorac Cardiovasc Surg. 1993; 106:116-25

3. Lundar T, Langmoen IA, Hovind KH. Fatal cardiopulmonary complications in children treated with ventriculoatrial shunts. Childs Nerv Syst. 1991;7:215-17.

4. Ryan US. The endothelial surface and responses to injury. Fed Proc. 1986;45:101-8.

5. Lambert V, Durand P, Devictor D, Planche C, Serraf A. Unilateral right pulmonary thromboendarterectomy for chronic embolism: a successful procedure in an infant. J Thorac Cardiovasc Surg. 1999;118:953-4. 